\title{
Decay of visual adaptation to tilt and displacement
}

\author{
GORDON M. REDDING \\ Illinois State University, Normal, Illinois 61761
}

\begin{abstract}
Persistence in the dark following $48 \mathrm{~min}$ of visual adaptation to tilt and displacement was compared in two experiments to determine if same or different processes are involved in the two kinds of adaptation. Decay of tilt adaptation occurred rapidly, all within about $16 \mathrm{~min}$. However, it was not complete and some residual tilt adaptation persisted for at least as long as $56 \mathrm{~min}$. Decay of displacement adaptation occurred more slowly but was clearly complete after at most $56 \mathrm{~min}$ in the dark. Displacement adaptation appears to be entirely subject to decay, while tilt adaptation involves an additional, more long-term component. Results are interpreted in terms of independent systems for the perception of location and orientation.
\end{abstract}

Few attempts have been made to experimentally compalte various kinds of perceptual adaptation (e.g.. Hajos \& Ritter, 1965; Pick \& Hay, 1964; Redding, 1973a) despite the fact that theories of adaptation (c.g.. Held, 1961; Rock, 1966) implicitly assume adaptation to a variety of optical transforms to be explicable within al single theoretical framework. Moreover, most of these studies have been concerned with parameters affecting the acquisition of aldiptation and little data are available relevant to the relationship between decay of tilt and displacement adaptation.

Some indirect comparisons are possible based on studies which have been concerned exclusively with one or the other transform. Hamilton and Bossom $(1464)$ failed to find a significant difference between decaly (during a dark period) and readaptation (during a period of exposure to the normal. untransformed world) after exposure to optical displacement. Ebenholtz (1968), however, found that following exposure to optical tilt readaptation proceeds more rapidly than does decay. These results suggest that displacement adaptation is entirely susceptible to decay while tilt adaptation involves a more persistent component which requires relearning for complete recovery of the normal state. This conclusion is supported by a later Ebenholtz (1969) study in which decaly of tilt adaptation was found to asymptote at a nonzero value. Similar data on decay of displacement adaptation are not available.

In a direct comparison of tilt and displacement adaptation, Redding (1973a) replicated the Ebenholtz hinding of asymptotic tilt decay and provided some information on displacement decay. Decay of tilt adaptation was not complete after $32 \mathrm{~min}$ in the dark

Thin study was supported by Research Grant MH 24420-01 from the National Institute of Mental Health. The author wishes to thank Gary W. Bradshaw, whose skill and dedication in data collection is comparable only with the patience of the students who participated as subjects in the experiments. Requests for reprints shoukl be sent to Gordon M. Redding. Department of Psychology. Illinois State University, Normal. Illinois 61761. and the decay that did occur was rapid, with substantially all decay occurring within the first $8 \mathrm{~min}$ of darkness and no further decrease thereafter. Displacement decay occurred more slowly and was continuous across the 32-min dark period. The titted functions suggested that displacement decay would reach zero, given enough time in the dark. These data suggest a major difference between the two kinds of adaptation. However, this conclusion must be qualified by the fact that the dark period was not long enough for displacement decay to reach a stable level. The present experiments were designed to complete this comparison of the two kinds of adaptation by extending the decay period. Tilt and displacement adaptation were investigated, respectively, in Experiments I and II.

\section{EXPERIMENT I}

In addition to extending the decay period beyond that previously studied. magnitude of optical tilt was also manipulated in the first experiment in order to determine the effects of this variable on decay of tilt adaptation. Ebenholtz (1969) produced evidence which suggests that rate of decay of visual compensation for optical tilt is independent of the level of adaptation at the start of the decay period. No significant difference was found between groups that began a 50 -min period in the dark at levels of adaptation ranging from approximately $2^{\circ}$ to $8^{\circ}$. However, the three groups of Ebenholtz differed in exposure conditions. The effects of such factors as exposure time and magnitude of tilt on rate of decay have not been systematically investigated.

Also, in Experiment 1, adaptation was measured in both the exposed and unexposed eyes in order to allow assessment of interocular transfer. Failure to find complete interocular transfer can be interpreted as indicating that the kind of adaptation under consideration does not involve the oculomotor system, but may. for example. involve local retinal cortical areas (Ebenholtz, 1970). Redding (1973a) consistently 
found adaptation to be less in the unexposed eye. the difference between eyes being significant in tilt decay but not in acquisition. Furthermore, an Eye by Exposure Time interaction appeared in displacement adaptation, with the unexposed eye approaching asymptote more rapidly than did the exposed eve. Since these previous data suggested that exposed vs. unexposed eye was likely to be a significant source of variance in both tilt and displacement adaptation. this variable was controlled in the present experiment.

\section{Method}

Procedure. During exposure, subjects walked through hallways while viewing an optically rotated visual field with the right eye, the left eye being occluded. Tilt was produced by a pair of dove prisms mounted in tandem and aftixed by a headset over the right eye. The dove prisms could be rotated to produce any desired degree of tilt up to $180^{\circ}$. and the visual field given by the prisms was approximately $10^{\circ}$ in diam.

Prior to the start of the adaptation period, each subject was tested on visual orientation by setting a luminous line $130 \mathrm{~cm}$ long. $.40 \mathrm{~cm}$ wide) to look lined up with his chin-forehead axis. Measurements were made to the nearest half-degree of rotation from objective vertical. All testing was conducted without prisms. with subjects viewing the line monocularly in a dark room at a distance of $121.92 \mathrm{~cm}$. The bottom of the line was the pivot point and was at eye level. on line with a point midway between the eyes. Both eyes were tested separately, and the head was held stationary by a forehead- and chinrest. In the preadaptation tests, the subjects made eight judgments, with the starting position of the line alternately at $25^{\circ}$ clockwise $(\mathrm{CW})$ and $25^{\circ}$ counterclockwise (CCW) of objective vertical. Right and left eyes were alternated every two judgments. Subsequent postadaptation tests involved only four judgments. alternating starting position of the line every judgment and eye tested every two judgments. Level of adaptation (LA) was detined separately for exposed (right) and unexposed (left) eyes as the difference between the mean of the last two preadaptation judgments (the first two judgments were considered practice and were dropped) and the mean obtained for each eye on subsequent tests. During exposure, tests were conducted at $12-\mathrm{min}$ intervals, four tests in all for a total exposure time of $\mathbf{4 8} \mathrm{min}$. Following the last adaptation test. the subjects continued to sit in the dark and seven decay tests were made at 8 -min intervals, for a total decay period of $56 \mathrm{~min}$.

To facilitate monocular testing, a circular shield was pivoted on the forehead crossbar of the forehead-and chinrest. The subjects were instructed, on alternate tests, to rotate the shield until it rested firmly against the nose on the side of the nontested eye. During all tests, taped white noise was played to mask any auditory cues from the operation of the measurement apparatus.

No attempt was made to identify the exact nature of the adaptation involved (e.g., proprioceptive change in felt eye position vs. local-sign change in retinal significance). However, several steps were taken to minimize the possibility of changes which have no general consequence for visual perception (i.e., changes other than those that may occur within the eye-head visual system). During exposure, the subjects were encouraged to move about freely in the halls, but were instructed not to look at any part of their bodies (particularly hands and feet) and not to touch walls. As a double precaution. the subjects wore a black cloak which covered the entire body from neck to knee. Thus. it is unlikely that any proprioceptive change, such as in felt position of the hand (Harris, 1963), or specific visual-motor compensation, such as eye-hand coordination (Mikaelian, 1967), occurred. By the use of egocentric (relative to the head) test instructions, it may be expected that, since the head

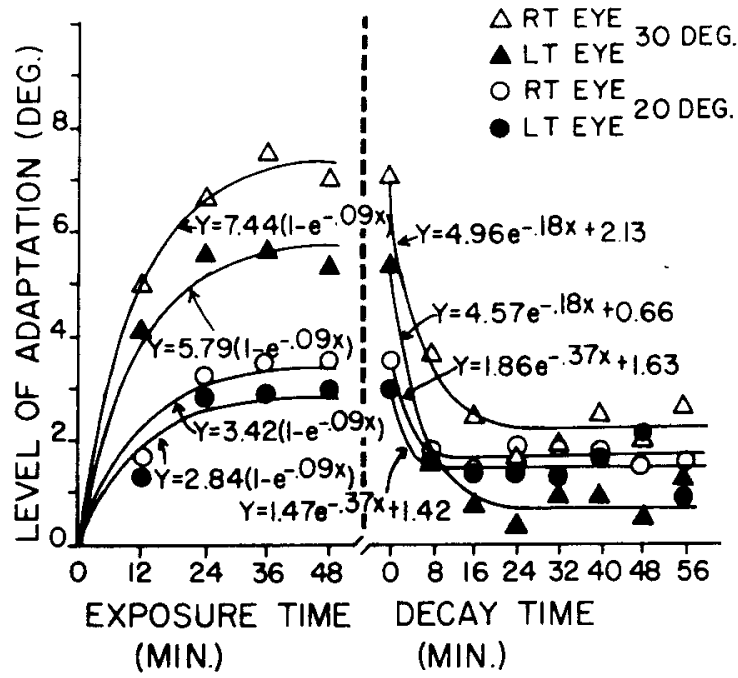

Figure 1. Mean level of adaptation to $30^{\circ}$ and $20^{\circ}$ of optical tilt in the exposed (right) and unexposed (left) eyes as a function of exposure time in acquisition and decay time in the dark. (Smooth curves represent the best fit by the method of least squares; Experiment 1.)

was always normally oriented in test, any abnormal proprioception would not influence the adaptation measure. The net effect of these specitications is to restrict consideration to the eye-head system. where adaptive change alters the phenomenal appearance of the visual world. To the extent that precautions were adequate, the adaptation measures may be assumed to reflect only changes in the visual system.

Design. Sixteen undergraduates (10 temale and 6 male) were run in two groups of 8 subjects each. The subjects were assigned alternately to one of the two groups as they arrived for experimentation. Two subjects, one in each group, failed to show at least $1^{\circ}$ of adaptation in either eye after $48 \mathrm{~min}$ exposure and were replaced. Rejected subjects were replaced by the next available subject such that alternate assignment to groups was maintained. The groups differed only in the magnitude of tilt received: $20^{\circ} \mathrm{CW}$ or $30^{\circ} \mathrm{CW}$. Counterbalancing within groups was complete for order in which eyes were tested (exposed eye or unexposed eye tirst), and order of starting position of the test line (CW or CCW first).

\section{Results and Discussion}

Separate analyses of variance were performed on adaptation scores obtained during exposure and dark periods, and the data were examined to determine the time course of acquisition and decay of tilt adaptation.

Acquisition. Since the control variable of starting position of the line was not found to be a signiticant source of main effect or interaction in preliminary analysis, the data were combined over this variable and a four-way analysis of variance performed. Significant effects were found for magnitude $[F(1,12)$ $=12.55, \mathrm{p}<.005]$, eye $[\mathrm{F}(1.12)=11.87, \mathrm{p}<.005]$, time $[F(3,36)=7.44, p<.001]$, and the Eye Order by Eye interaction $[\mathrm{F}(1,12)=6.95, \mathrm{p}<.025]$. Figure 1 represents $L A$ as a function of exposure time for two levels of optical tilt and for exposed (right) vs. unexposed (left) eyes. The smoothed curves represent negatively accelerated exponential growth functions 
litted to the data by the method of least squares. The fitted functions have the form $L A=a\left(1-e^{-b t}\right)$, where $a$ is the estimated asymptote of adaptation and b estimates the rate at which adaptation approaches the asymptote as a function of time, $t$. Since there nere no signilicant interactions with time, rate of adaptation was assumed to be independent of transform magnitude and eye tested, and a single rate parameter $(.09)$ was estimated for the data points. The functions employing tive parameters for 16 data points tit the data reasonably well, yielding a standard error of estimate ${ }^{1}$ of .30 .

Adaptation was greater to $30^{\circ}(\overline{\mathrm{X}}=5.83)$ than to $20(\bar{X}=2.71)$ of tilt. Adaptation was also greater in the exposed eye $(\bar{X}=4.75)$ than in the unexposed eye $(\bar{X}=3.79)$. Although the exposed eye was always numerically greater in adaptation, the difference between eyes wats smaller when the unexposed eye was tested first ( $\bar{X}=4.23$ for the exposed eye and $\bar{X}=$ 4.00 for the unexposed eye) than when the exposed eye was tested first ( $\bar{X}=5.28$ for the exposed eye and $\bar{X}$ $=3.58$ for the unexposed eye). Thus, the obtained interocular difference appears to be largely due to an advantage enjoyed by the exposed eye when it is tested tirst. In light of previous failures to find significant differences between exposed and unexposed eyes (Mack \& Chitayat, 1970; Quinlan, 1970; Redding, 1973a). the present interocular differences must be interpreted calutiously. Typically, numerical equivalence between exposed and unexposed eyes has been found in studies which have counterbalanced exposure of both eyes (Mack \& Chitayat, 1970; Quinlan, 1970), while studies which have found greater (but usually nonsignificant) adaptation in the exposed eye have not employed such counterbalancing. usually restricting exposure to the right eye (Ebenholt\%. 1966: Redding. 1973a). It seems likely that the present difference beween eyes was due to this asymmetry of exposure conditions.

The finding of a main effect for exposure time is virtually unique in studies of tilt adaptation, where adaptation is usually asymptotic at the first test and shows little change thereafter (e.g.. Ebenholtz, 1966). Redding (1973a) tested for tilt adaptation as early as 15 min. linding no significant further increase at subsequent tests after 30 and $45 \mathrm{~min}$. In contrast. subsequent orthogonal comparisons of the present data indicate that adaptation continues to increase up to the second test and is unchanged thereafter. Adaptation at $12 \mathrm{~min}(\overline{\mathrm{X}}=3.02)$ is significantly less than the average found at $24 \mathrm{~min}(\bar{X}=4.55)$. 36 min $(\bar{X}=4.84)$, and $48 \mathrm{~min}(\bar{X}=4.67)$ $[F(1.36)=21.93, p<.001]$. Adaptation at $24 \mathrm{~min}$ is not different from the average adaptation found at 36 and $48 \mathrm{~min}[\mathrm{~F}(1.36)=.34]$. Considering these results, together with those of Redding (1973a), leads to the conclusion that the limit of tilt adaptation is reached between 12 and $15 \mathrm{~min}$ of exposure. Tilt adaptation grows rapidly up to this point, then levels off and little or no further increase occurs.

Decay. Figure 1 also shows LA for the exposed and unexposed eyes at eight decay tests for the two levels of optical tilt. The last exposure test at $48 \mathrm{~min}$ was taken as the starting LA, zero time, for which to measure decay. The smoothed curves represent negatively decelerated exponential functions fit to the datal by the method of least squares. Preliminary analysis indicated that neither order of starting position nor order in which the two eyes were tested were significant sources of variance and neither were signiticant interactions associated with these control variables. Consequently, the data were combined for a three-way analysis of variance. Significant sources of variance for tilt decay were eye $[F(1,14)=6.93$, $p<.0251$, time $[F(7.98)=13.51, p<.001]$, and the Magnitude by Time interaction $[F(7,98)=3.70$, $p<.005]$. Trend analysis indicated that $20^{\circ}$ and $30^{\circ}$ of tilt did not differ in the linear component of decay $[F(1,14)=2.76]$, but the two groups did differ in the quadratic component $[\mathrm{F}(1,14)=14.67, \mathrm{p}<.005]$. The results of the trend analysis reflect the fact, apparent in Figure 1, that decay was rapid for the $20^{\circ}$ group, reaching a stable level by the first decay test, while the $30^{\circ}$ group showed somewhat slower decaly, not stabilizing until at least the second decay test at 16 min. Consistent with this interpretation of the interaction, the data for both $20^{\circ}$ and $30^{\circ}$ were fit with the same function. The rate parameter was allowed to vary to reflect the Magnitude by Time interaction. The titted functions have the form LA = $a e^{-b t}+c$, where $a+c$ is the LA at the beginning of the decaly period, $b$ indicates the rate of decay per unit of time, $t$, and $c$ is the asymptote of decay. The standard error of estimate for the functions employing 10 parameters for 32 data points was .28 .

The presence of interocular differences in decay, as in acquisition. may be presumed to reflect the asymmetry in the experimental design rather than incomplete transfer. Assuming that the fitted functions accurately reflect the time course of decay, several conclusions are possible concerning tilt adaptation. Decay of adaptation to $20^{\circ}$ of tilt is much faster, approaching asymptote more quickly, than that for $30^{\circ}$ of tilt, as indicated by the Magnitude by Time interaction and reflected in the larger rate parameter estimated for the $20^{\circ}$ group (.37) than for the $30^{\circ}$ group (.18). However, decay is not complete for either group. The estimated asymptotes for the fitted functions shown in Figure 1 are all greater than zero, and the data points themselves are all signiticantly greater than zero $(95 \%$ confidence limits) atter as much as $56 \mathrm{~min}$ in the dark for all conditions except the unexposed eye in the $30^{\circ}$ group. The confidence limits for this latter condition include zero at all tests after $8 \mathrm{~min}$. However, this low level of adaptation is due almost entirely to one 
subject. who showed unusually large negative values in his left eye. The fact that virtually all decay occurs within the first $16 \mathrm{~min}$, with little or no further decrease up to $56 \mathrm{~min}$ in the dark, suggests that some residual tilt adaptation persists indefinitely unless the opportunity for relearning occurs.

\section{EXPERIMENT II}

The second experiment investigated displacement adaptation and was designed to be the analog for the study of tilt adaptation in Experiment I. The same exposure and decay times were used, displacement magnitude was manipulated, and both eyes were tested.

\section{Method}

The method of Experiment II differed in several ways from that used in Experiment 1. Displacement was produced by variable Risley prisms set in a goggle frame over the right eye. The Risley prism could be varied to produce any desired amount of displacement up to $30 \mathrm{D}$, and the visual field given by the prisms was approximately $20^{\circ}$ in diam. Visual direction was tested by having the subject set a luminous dot $(.25 \mathrm{~cm}$ diam) to look straight ahead of his nose in the horizontal plane. Measurement was to the nearest $1 / 2$-arc-deg deviation from objective straight ahead. The subjects viewed the dot monocularly in a dark room. The dot was at eye level and moved along an are such that a constant distance of $121.92 \mathrm{~cm}$ from the subject was maintained. Starting positions of the dot in test were 8 arc deg right (R) and left (L) of objective straight ahead.

Pilot work suggested that the presence of the shield resting against the nose caused the subjects to consciously compensate in their judgments of straight ahead for the lateral positioning of the eye relative to the nose. When the right eye is tested, the subject tended to move the dot to the left of what looked straight ahead, while the opposite compensation was made when the left eye was tested. In order to minimize such contamination of judgments by conscious correction, the shield used in Experiment I was replaced with an eye patch which the subject shifted from eye to eye and which was less prone to draw attention to the nose.

Initially, two groups of 8 subjects each were run using the same rejection criterion as in Experiment $I$, i.e., the subjects were replaced if they did not show at least $1^{\circ}$ of adaptation in both eyes after $48 \mathrm{~min}$ of exposure to displacement. With this criterion, only 3 subjects receiving $30 \mathrm{D}$ of displacement were rejected, while 10 subjects receiving $20 \mathrm{D}$ were rejected. In order to eliminate the obvious bias in favor of higher adaptors in the 20-D group, a third group was run receiving $20 \mathrm{D}$ of displacement. A total of 12 subjects were run in this third group, with the 3 subjects showing the lowest LA after $\mathbf{4 8}$ min exposure being dropped in order to minimize any sampling differences compared to the original 30-D group. Additionally, one extremely deviant subject was replaced who, during the dark period, showed increasing adaptation up to five times the level obtained at the end of exposure. The data on the remaining 8 subjects receiving $20 \mathrm{D}$ were then combined for analysis with those from the original 30-D group, for a total of 16 subjects (7 female and 9 male). As in Experiment $I$, counterbalancing within groups was complete for order in which the eyes were tested (right or left first) and order of starting position of the test dot ( $R$ or $L$ first). In all other respects, the method was similar to that of Experiment $\mathrm{I}$.

\section{Results and Discussion}

Separate analyses of variance were performed on adaptation scores obtained during exposure and dark perinds. and the datal were examined to determine the time course of acquisition and decay of displacement adaptation.

Acquisition. Preliminary analysis indicated that starting position was not a significant main effect or a source of interation. The data were, therefore, combined over starting positions and subjected to a four-way analysis of variance. Adaptation was signilicantly greater $[\mathrm{F}(1.12=4.77, \mathrm{p}<.05]$ to 30 - $\mathrm{D}$ displacement $(\overline{\mathrm{X}}=5.38)$ than to $20-\mathrm{D}$ $(\bar{X}=3.08)$. however. the difference between groups is qualitied by the presence of a Magnitude by Eye order interaction $[F(1.12)=6.49, p<.05]$. The nature of the interaction is such that a substantial difference between groups occurred when the left eye wals tested tirst. When the right eye was tested first, this difference did not appear. An explanation of this effect is not immediately available, and it seems probable that the interaction reflects sampling error.

No signiticant difference appeared between eyes $[F(1,12)=.05]$. The mean LA for the unexposed eye was higher than for the exposed eye in the 30-D group (5.15 and 5.62 for exposed and unexposed eyes, respectively), while the reverse was true for the 20-D group (3.44 and 2.73 for exposed and unexposed eyes, respectively). However, the Magnitude by Eye interaction was not significant $[F(1,12)=1.31]$. Thus, the obtained interocular differences may be interpreted as chance occurrences, and, consistent with previous lindings (Hajos \& Ritter, 1965; Redding, 1973a), the present results may be taken to indicate that interocular transfer is complete for displacement adaptation. The fact that the second eye tested suffers some loss of adaptation due to delay of test is reflected in the significant Eye by Eye Order interaction $[\mathrm{F} 1,12)=6.70, \mathrm{p}<.025]$.

The only other significant source of variance in displacement acquisition was exposure time $[F(3,36)$ $=3.77, \mathrm{p}<.025]$. Figure 2 shows LA as a function of exposure time for two levels of displacement and for the exposed and unexposed eyes. As in Experiment I, the smoothed curves represent negatively accelerated exponential growth functions tit to the data by the method of least squares. Since exposure time did not interact with any other variable, rate of adaptation was assumed independent of transform magnitude and eye tested, and a single rate parameter was estimated (.06) for the 16 data points. The asymptote parameters were allowed to vary to reflect the numerical differences among the conditions. The curves fit the data reasonably well for descriptive purposes, yielding a standard error of estimate of .74 for functions employing five parameters for 16 data points.

Assuming that the fitted functions accurately represent the acquisition of adaptation, several contrasts between tilt and displacement are possible. The estimated rate parameter is somewhat smaller for displacement adaptation (.06) than for tilt adaptation 


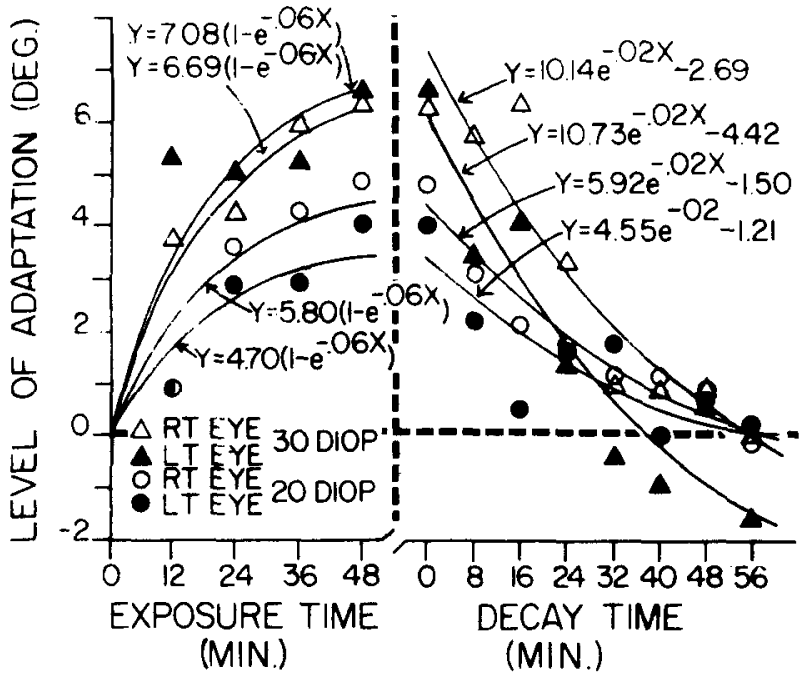

Figure 2. Mean level of adaptation to 30 and $20 \mathrm{D}$ of optical displacement in the exposed (right) and unexposed (left) eyes as a function of exposure time in acquisition and decay time in the dark. (Smooth curves represent the best fit by the method of least squares; Experiment II.)

(.)9, see Figure 1), indicating a slower rate of growth for displacement. Although the absolute amount of adaptalion differs, the present difference in rate of growth for tilt and displacement is in the same direction as that found in previous work. For cxample, in Experinent 1 of Redding (1973a) the estimated rate parameter for displacement was .06 , same as in the present study. The rate parameter for tilt was much larger at .19 . The slower rate of tilt adaptation found in the present study is probably due to the fact that Redding (1973a) did not measure tilt adaptation carlier than $15 \mathrm{~min}$, at which time tilt adaptation is already asymptotic. Thus, it may be concluded. consistent with previous work, that displacement adaptation occurs more slowly than does tilt adaptation.

Decay. Also represented in Figure 2 is the LA for the exposed and unexposed eyes at eight decay tests for the two levels of displacement. The smoothed curves represent negatively decelerated exponential functions lit to the data by the method of least squres. Neither statting position nor eye order were signilicant sources of main effects or interactions, and the data were combined for a three-way analysis of variance. $A s$ was the case in acquisition. the difference in LA between exposed $(\bar{X}=2.48)$ and unexposed eyes $(\bar{X}=1.54)$ was not significant $[F(1,14)=2.70 \mid$, indicating that decay, as well as acquisition, of displacement adaptation is comparable in the two eyes. Decay time was the only other signiticant source of variance $[F(7,98)=7.90$, $\mathrm{p}<.0(011$, and trend analysis revealed only a signilicant linear component $[\mathrm{F}(1,14)=11.66$, $p<.005\}$. Since linear decay seems unlikely and in order to allow comparison with tilt decay, the quadratic functions used in Experiment I were fit to the displacenent data. A single rate parameter (.02) was estimated since the Magnitude by Time interation was not significant, $F(7,98)=1.53$. Although the difference between magnitudes was not signilicant $[F(1,14)=.33]$, the other parameters were allowed to vary in order to provide a better description of the numerical data. The functions fit the data reasonably well for descriptive purposes, yielding a standard error of estimate of .76 for functions employing nine parameters for 32 data points.

Clearly, displacement adaptation decays completely in the dark. The estimated asymptotes for all conditions are less than zero, and the data points themselves are all at or below zero by $56 \mathrm{~min}$ in the dark. The unexposed eye for the 30-D group shows substantial negative values as early as $32 \mathrm{~min}$. However, this is due almost entirely to one deviant subject. who showed unusually large negative values in his left eye, particularly at 40- and 56-min tests. Decay of displacement adaptation also occurs relatively slowly, as reflected by the small rate parameter (.02) estimated for the two displacement magnitudes, and the rate of displacement decay appears to be independent of LA at the beginning of the decay period. However, this last conclusion must be qualified by the fact that the difference between groups was small in acquisition and nonsignificant in decaly. Larger differences in LA at the beginning of the decay period might reveal differences in the rate of displacement decay as a function of initial LA.

\section{CONCLUSION}

Assuming that the curves in Figures 1 and 2 accurately represent the time course of decay, several differences between tilt and displacement adaptation are obvious. Decay of displacement adaptation occurs much more slowly than does tilt adaptation. This difference is reflected by the fact that the estimated rate parameters for tilt are both much larger (.18 and .37 for $20^{\circ}$ and $30^{\circ}$ tilt, respectively) than the single rate parameter estimated for the two magnitudes of displacement (.02). Another difference between the two transforms would seem to be that tilt adaptation decays more rapidly the greater the initial LA, while the rate of displacement decay is independent of LA at the beginning of the decay period. However, this conclusion is qualitied by the relatively small initial difference between groups receiving different magnitudes of displacement. Finally, displacement adiatation is entirely subject to decay. Displacement deciay occurs relatively slowly, but is clearly complete after at most $56 \mathrm{~min}$ in the dark. In contrast, tilt adaptation involves a more persistent component which is not subject to decay. Tilt decay occurs rapidly. all within at most $16 \mathrm{~min}$ of darkness; 
however. it is not complete and some residual adaptation effect persists for at least as long as $56 \mathrm{~min}$ of darkness. It seems not unreasonable to conclude that tilt decay is asymptotic short of complete decay after approximately $16 \mathrm{~min}$ in the dark.

The present memorial differences between tilt and displacement adaptation are consistent with other parametric differences between the two kinds of adaptation (Redding, 1973a) and indicate that different systems are involved in adaptation to the two transforms. Furthermore, Redding (1973b) found that adaptation to both transforms presented simultaneously is not more difficult than adaptation to each transform separately. The lack of interference when the two transforms are presented simultaneously argues for separate and independent mechanisms mediating adaptation to optical tilt and displacement. Displacement adaptation may be assumed to implicate the mechanisms responsible for the perception of visual direction, which requires that eye position be taken into account, while tilt adaptation involves the relational analysis necessary for the perception of form. The transitory nature of displacement adaptation may be related to the relatively greater lability of visual direction in the absence of a visual frame of reference (e.g., autokinetic movement). If, as seems likely, displacement adaptation consists in a change in registered eye position (Ebenholtz, 1970; Harris, Note 2), the memorial differences from tilt adaptation may have their origin in a greater lability for eye position calibration compared to the relative stability of the relational framework within which visual orientation is evaluated.

\section{REFERENCES}

Ebenholtz, S. M. Adaptation to a rotated visual field as a function of degree of optical tilt and exposure time. Journal of Experimental Psychology, 1966, 72, 629-634.

Ebenholtz, S. M. Some evidence for a comparator in adaptation to optical tilt. Joumal of Experimental Psychology, 1968 , 77. $94-100$
Ebenholtz, S. M. Readaptation and decay after exposure to optical tilt. Joumal of Experimental Psychology, 1968, 78, 350-351.

Ebenholtz. S. M. Transfer and decay functions in adaptation to optical tilt. Joumal of Experimental Psychology, 1969, 81, 170-173.

Ebenholtz, S. M. On the relation between interocular transfer of adaptation and Hering's law of equal innervation. Psychological Review, 1970, 77, 343-347.

Hamilton, C. R., \& Bossom, J. Decay of prism aftereffects. Journal of Experimental Psychology, 1964, 67, 148-150.

Hajos, A., \& Ritter, M. Experiments to problems of interocular transfer. Acta Psychologica, 1965, 24, 81-90.

HARRIS. C. S. Adaptation to displaced vision: Visual, motor, or proprioceptive change? Science, 1963, 140, 812-813.

HELD, R. Exposure-history as a factor in maintaining stability of perception and coordination. Journal of Nervous and Mental discase, 1961, 132, 27-32.

Mack, A., \& Chitayat, D. Eye-dependent and disparity adaptation to opposite visual-field rotations. American Journal of Psychology, 1970. 83, 352-371.

Mikaelian, H. H. Relation between adaptation to rearrangement and the source of motor-sensory feedback. Psychonomic Science, $1967,9,485-486$.

Pick, H. L., \& Hay, J. C. Adaptation to prismatic distortion. Psychonomic Science, 1964, 1, 199-200.

Quinlan, D. Effects of sight of the body and active locomotion in perceptual adaptation. Joumal of Experimental Psychology, $1970,86,91-96$.

RedDing, G. M. Visual adaptation to tilt and displacement: Same or different processes? Perception \& Psychophysics, 1973, 14, 193-200. (a)

Redding, G. M. Simultaneous visual adaptation to tilt and displacement: A test of independent processes. Perception \& Psychophysics, 1973, 2. 41-42. (b)

Rock, I. The nature of perceptual adaptation. New York: Basic Books, 1966.

\section{NOTES}

1. The standard error of estimate is defined as the square root of the averaged square deviation of obtained from predicted LA.

2. Harris, C. S. Through the looking glass: Adapting to an optically reversed world. Symposium on Perceptual Change, American Association for the Advancement of Science, Washington, D.C., December 1966.

(Received for publication August 7, 1974; revision received November 5,1974 .) 